\title{
Modeling the Effects of Vaccination on Chronically Infected HIV-Positive Patients
}

\author{
*Laura E. Jones and †Alan S. Perelson \\ *Ecology and Evolutionary Biology, Cornell University, Ithaca, New York; and †Theoretical Biology and Biophysics Group, Los \\ Alamos National Laboratory, Los Alamos, New Mexico, U.S.A.
}

\begin{abstract}
T-cell activation plays a critical role in the initiation and propagation of HIV-1 infection and yet transient activation of the immune system is a normal response to immunization. While it is now considered wise to vaccinate HIV-1-positive patients, it is crucial to anticipate any lasting effects of vaccination on plasma HIV-1 RNA levels and on infected T-cell populations. We extend a simple dynamic model of HIV infection to include T-cell activation by vaccination. We show that the model can reproduce many but not all of the features of the post-tetanus immunization rise in viral load observed and reported on by Stanley et al. in 1966 ( $N$ Engl J Med, 334:12221230). Amplitudes and approximate timing of postimmunization peak viral loads were matched in 10 of 12 cases; in patients with double postimmunization peaks of nearly equal amplitude the later peaks were matched. Furthermore, our simulations suggest that productively infected cell populations track postvaccination increases in plasma viral load, rising and falling in concert over a period of about 4 weeks, whereas chronically infected cells peak later and remain elevated over baseline levels for up to 6 weeks postvaccination. Key Words: Viral dynamics-Mathematical modelsTetanus vaccination.
\end{abstract}

T-cell activation plays a critical role in HIV infection and progression to AIDS (1-4). In vitro studies have demonstrated the importance of cell activation in establishing productive HIV infection. For example, reverse transcription within resting cells may be incomplete and integration of proviral DNA may not occur, resulting in abortive infection $(5,6)$. In contrast, activated peripheral blood mononuclear cells are readily infected in culture (5-7). The course of HIV infection in vivo appears to be influenced by cell activation. High levels of activated peripheral $\mathrm{T}$ cells are a predictor of early progression to AIDS $(8,9)$, and chronic immune activation resulting from frequent and repeated parasitic infection has been

This work was performed under the auspices of the U.S. Department of Energy and was supported by NIH grants AI28433 and RR06555.

Address correspondence and reprint requests to Alan S. Perelson, Theoretical Biology and Biophysics, MS-K710, Los Alamos National Laboratory, Los Alamos, NM 87545, USA; e-mail: asp@lanl. gov

Manuscript received February 28, 2002; accepted September 10, 2002. suggested as the probable cause of the increased rate of HIV progression to AIDS and the greater susceptibility to infection observed in sub-Saharan Africa compared with the developed countries (10-12).

Transient activation of the immune system occurs during infections and is a normal response to immunization. Such activation can affect the course of HIV infection. Numerous studies have examined the consequences of vaccinating both untreated (2,13-18) and treated HIVinfected individuals (18-21). Stanley et al. (2) found that giving a booster dose of tetanus toxoid resulted in transient increases in plasma viremia in all $13 \mathrm{HIV}$-infected subjects studied. Staprans et al. (13) found that influenza vaccination led to transient increases of plasma HIV-1 RNA, and that patients with higher CD4 T-cell counts had larger and more rapid increases in viral load. The large immunization-related increases in virus reported by Staprans et al. (13) suggest that the observed viral replication may be correlated with activation of both antigen-specific T-cells and other non-specific T-cell populations, which, once activated, then become targets for infection (3). In a double-blind placebo-controlled study of the effects of influenza vaccination on HIV-infected 
adults, both untreated and on monotherapy, Tasker et al. (18) found statistically significant increases in plasma viral load in the vaccine group relative to placebo recipients at one month postvaccination.

Studies using influenza vaccination and pneumococcal vaccine have confirmed that vaccination can trigger increases in plasma HIV RNA (14-21). Thus a number of data sets are available that illustrate a relationship between immune system activation and changes in HIV viral load. This data presents an interesting challenge to current mathematical models of HIV infection (22-24). In an early theoretical paper, McLean and Nowak (25) examined the effects of infection with pathogens other than HIV as a cofactor in HIV progression. However, no quantitative data was available at the time for direct comparison of model and theory. A more recent study by Ferguson et al. (26) presented a model and some comparisons with the average change in plasma HIV-1 RNA seen in the Stanley et al. (2) study for vaccination of HIV-1-infected untreated patients, but concentrated primarily on antigen-driven T-cell proliferation and residual viral replication for patients on antiretroviral therapy. Here we focus on the effects of vaccination on untreated HIV-infected patients and analyze the same data as Ferguson et al. (23) (i.e., the Stanley et al. (2) dataset) but on a patient-by-patient basis. We construct a simple model of HIV-infection and immune system activation via vaccination, and then compare the model with data from the study of Stanley et al. (2) in which 13 HIV seropositive, asymptomatic individuals were vaccinated with tetanus toxoid.

\section{A Simple Vaccination Model}

To understand the effects of vaccination with a common recall antigen (tetanus toxoid) on chronically infected, untreated HIV-infected patients whose viral loads have reached steady state, we modify what has become a standard HIV infection model (23-24). The model includes uninfected, infected and chronically infected Tcell populations, HIV, and other antigens, A. The model omits latently infected cells because in untreated individuals they are present at very low levels compared with actively infected and chronically infected cells; Chun and Siliciano (27) estimate that only 1 in $10^{5} \mathrm{~T}$ cells are latently infected. Note that in patients receiving potent antiretroviral therapy, a recent study suggests that mobilization of the latently infected cell pool may occur postvaccination (19). We also do not separately consider vaccine-specific or HIV specific T-cell populations, because this leads to a model with more parameters than the data reported by Stanley et al. (2) will support.
The model, like that of McLean and Nowak (25), includes complexities of the antigen-specific, helper celldependent response that leads to antigen elimination. Because individuals would be expected to vary with regard to the time of their last tetanus vaccination, one might assume that levels of antibody and $\mathrm{T}$ memory cells specific for tetanus toxoid would also vary. In our model we avoid this level of detail, but simply assume that the antigen clearance rate constant, $\gamma$, and parameters that determine the rate of $\mathrm{T}$ cell activation vary for each patient. The model we use is given by the following system of differential equations:

$$
\begin{aligned}
& \frac{d A}{d t}=-\gamma A T \\
& \frac{d T}{d t}=\lambda+a\left(\frac{A}{A+K}\right) T-d T-k V T \\
& \frac{d T^{*}}{d t}=(1-\alpha) k V T-\delta T^{*} \\
& \frac{d C}{d t}=\alpha k V T-\mu C \\
& \frac{d V}{d t}=N \delta T^{*}+N_{c} \mu C-c V
\end{aligned}
$$

where $A$ is the vaccine antigen, $T$ are uninfected $C D 4^{+} \mathrm{T}$ cells, $T^{*}$ are productively infected cells, $C$ are chronically infected cells, and $V$ represents HIV. Vaccine antigen is cleared in a T-cell-dependent manner with rate constant $\gamma$. Uninfected $\mathrm{T}$ cells, $T$, are produced at a rate $\lambda$, die at a rate $d$, and are infected by virus with rate constant $k$. In the presence of antigen, we assume T cells are activated into proliferation at a maximum rate $a$, and that the proliferation rate depends on the antigen concentration with a half-saturation constant $K . K$ is thus the antigen concentration that drives $\mathrm{T}$ cell proliferation to half its maximal value. Note that though we do not separate out antigen-specific $\mathrm{T}$ cells, the activation parameter, $a$, incorporates the fact that only a fraction of $\mathrm{T}$ cells may be activated by antigen. For example, if $1 \%$ of $\mathrm{T}$ cells are antigen-specific, then one might envision the second term on the right-hand side of equation 2 having $T$ replaced by $0.01 T$. If this were done, then the factor of 0.01 could be incorporated into the unknown parameter $a$, and the equation would remain as written.

Productively infected cells, $T^{*}$, are generated by infection of target $\mathrm{T}$ cells, $T$, at a rate $k V T$, and die at rate $\delta$, as in the standard model (23). Chronically infected T cells, $C$, are produced from susceptible $\mathrm{T}$ cells at a rate $\alpha k V T$, where $\alpha<1$. Thus, the production of chronically 
infected cells occurs at a fraction of the rate productively infected cells are generated. Chronically infected cells die at a rate $\mu$, which we assume is less than $\delta$, so that chronically infected cells are longer-lived than productively infected cells. Free virus, $V$, is produced by productively infected cells at average rate $N \delta$, by chronically infected cells at rate $N_{\mathrm{c}} \mu$, and is cleared at rate $c$ per virion. Based on previous work we assumed $\lambda=1 \times$ $10^{4} \mathrm{ml}^{-1}(28) ; d=0.01 d^{-1}(29) ; \delta=0.7 d^{-1}(22) ; \alpha=$ 0.195 (28); $\mu=0.07 d^{-1}(22) ; N_{\mathrm{c}}=4.11$ (28), and $c=$ $13 d^{-1}$ (26,30). Before vaccination, each patient was assumed to be in steady state with a known total baseline T-cell count, $\bar{T}_{T}$, and viral load, $\bar{V}$.

Given these measurements and the steady-state conditions derived from equations 2-5 (see Appendix), initial numbers of infected cells can be derived as well as a set of parameters describing chronic HIV infection for each patient.

At time $t=0$, we assume a dose of antigen, $A_{0}$, was given in a vaccine, which perturbed the steady state. We assume the same dose of vaccine was given to all individuals, and then model the antigen remaining in each individual as a fraction of the immunizing dose. This is equivalent to setting $A_{0}=1$. We numerically solve the system of differential equations given by $1-5$, with the initial conditions $A(0)=1$, and $T, T^{*}, C$, and $V$ set to their steady state values. For each individual studied, the parameters $K, \alpha$, and $\gamma$ were allowed to vary so that the amplitude and timing of the response could be best matched to the observed postvaccination viremia.

\section{Data and Sampling}

Stanley et al. (2) studied 16 asymptomatic homosexual men seropositive for HIV-1. Thirteen subjects were given a $0.5-\mathrm{mL}$ tetanus booster intramuscularly and three were mock immunized. After vaccination, viral load measurements were taken on days 0 (baseline), 4, 7, 14, 21,28 , and 42 . No information on prior vaccination history (i.e., date of last tetanus booster, or general quality of health) was given. It was noted that one patient developed cavitary pneumonia about a month after vaccination.

Of the 13 patients reported on by Stanley et al. (2), we did not include patient 13 in our study. Patient 13 had a baseline T-cell count of 8 cells $/ \mathrm{mm}^{3}$, which is too low to be consistent with our parameter assumptions and the prevaccination steady-state assumption of our model (see Appendix). Thus either the patient was not at true steady state, or the parameter values chosen from the literature are not applicable to this patient. The baseline characteristics for the remaining 12 patients are given in Table 1.

Data obtained for a subset of the patients are shown in Figure 1. The data sampling was too sparse to determine the exact peak of viremia or the time the peak was attained. However, Stanley et al. (2) report the apparent peak and the time it was measured. Many patients had two postimmunization peaks in viral load. The larger peak was the earlier of the two observed peaks in patients $3,5,10$, and the second of two peaks in patients $1,4,7$, 11 , and 12. Patients 2, 8, and 9 had two peaks of nearly equal amplitude (Fig. 1A).

Of the patients with double postimmunization peaks in viremia, patients $1,7,8,9$, and 11 had peaks at days 7 and 21 (Fig. 1A), though in some of these cases the earlier peak can be classified as a minor peak or an inflection point. These minor peaks might be the result of assay variation and hence were not considered significant. Of those with single observed postimmunization peaks, patients 3,5 , and 10 have primary peaks at day 7 , and inflection points, shoulders, or minor peaks at day 28 (Fig. 1B).

TABLE 1. Baseline characteristics and postimmunization plasma viremia

\begin{tabular}{|c|c|c|c|c|c|c|c|}
\hline Patient & $\begin{array}{l}\mathrm{T} \text { cells, } T_{T} \\
\left(\text { cells } / \mathrm{mm}^{3}\right)\end{array}$ & $\begin{array}{c}\bar{T} \\
\text { (cells/mm } / \mathrm{mm}^{3} \text { ) }\end{array}$ & $\begin{array}{c}\bar{T}^{*} \\
\left(\text { cells } / \mathrm{mm}^{3}\right)\end{array}$ & $\begin{array}{c}\bar{C} \\
\text { (cells } / \mathrm{mm}^{3} \text { ) }\end{array}$ & $\begin{array}{c}V_{0}^{a} \\
\text { (RNA/mL) }\end{array}$ & $\begin{array}{c}V_{p} \\
\text { (RNA/mL) }\end{array}$ & $V_{p} / V_{0}$ \\
\hline 1 & 362 & 336 & 7.6 & 18.5 & 147,000 & $437,000(21 / 20)^{b}$ & 2.97 \\
\hline 2 & 271 & 241 & 8.7 & 21.1 & 3850 & $10,500(14,28 / 14)$ & 2.73 \\
\hline 3 & 350 & 323 & 7.8 & 18.8 & 120,000 & $900,000(7 / 8)$ & 7.50 \\
\hline 4 & 389 & 364 & 7.3 & 17.7 & 100,000 & $700,000(4,21 / 18)$ & 7.00 \\
\hline 5 & 586 & 569 & 4.9 & 12.0 & 21,000 & $45,000(4,21 / 19)$ & 2.14 \\
\hline 6 & 336 & 309 & 7.9 & 19.3 & 215,000 & $725,000(7 / 9)$ & 3.37 \\
\hline 7 & 336 & 309 & 7.9 & 19.3 & 75,000 & $315,000(7,21 / 18)$ & 4.20 \\
\hline 8 & 361 & 335 & 7.6 & 18.5 & 87,500 & $241,000(7,21 / 19)$ & 2.75 \\
\hline 9 & 497 & 476 & 6.0 & 14.6 & 220,000 & $745,000(7,21 / 23)$ & 3.38 \\
\hline 10 & 615 & 599 & 4.6 & 11.1 & 10,500 & $375,000(7 / 7)$ & 35.7 \\
\hline 11 & 363 & 337 & 7.6 & 18.5 & 80,000 & $225,000(7,21 / 18)$ & 2.81 \\
\hline 12 & 403 & 378 & 7.1 & 17.3 & 7000 & $65,000(4,28 / 27)$ & 9.28 \\
\hline
\end{tabular}

${ }^{a} V_{0}=$ baseline viral load, $V_{p}=$ peak viremia.

${ }^{b}$ First number(s) are observed time(s); second number, modeled time to peak viremia (d). 

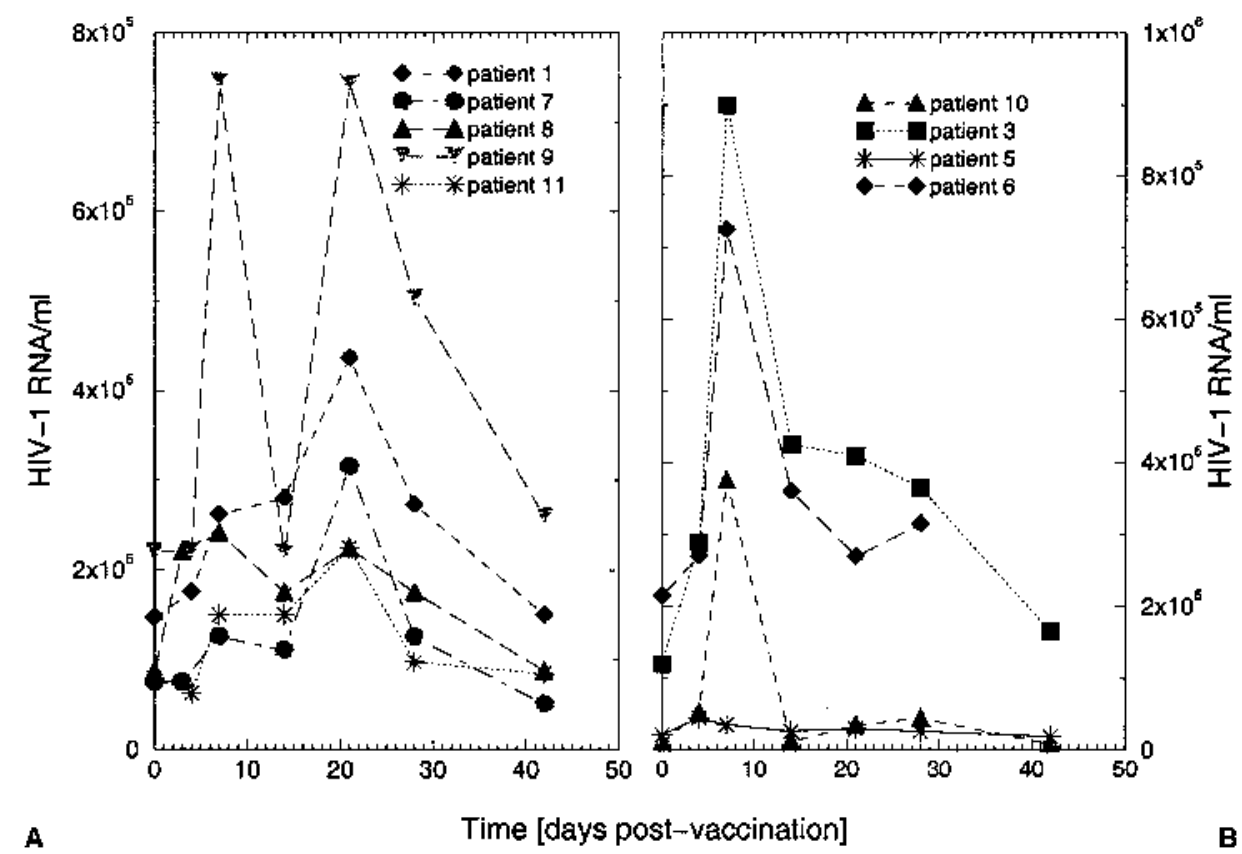

A

Time [days post-vaccination]

B

FIG. 1. Data from Stanley et al. (2) showing postvaccination rises in plasma HIV-1 RNA. (A) Patients experiencing two distinct peaks in viremia at roughly days 7 and 21. Patients 8 and 9 have equal or nearly equal peaks at days 7 and 21 , whereas patients 1,7 , and 11 experience what was recorded as a minor peak at day 7 followed by a true peak at day 21 . (B) Patients experiencing one early primary peak in viremia at day 7 . Note that the curves for most of these patients show a postpeak "shoulder" at days 21-28.

\section{RESULTS}

For each of the 11 patients studied, the model was fit to the data on viral load changes after vaccination. Using nonlinear regression techniques, the best-fit set of parameters was then determined for each patient (Table 2).

The amplitude and the approximate timing of the postimmunization maximum viremia were matched in 10 of 12 cases; in patients with double peaks of nearly equal amplitude (i.e., patients 8, 9), the later peaks were matched. The results are summarized in Tables 1 and 2 .

TABLE 2. Derived and best-fit parameter values for modeling postimmunization plasma viremia

\begin{tabular}{rcrlcr}
\hline $\begin{array}{c}\text { Patient } \\
\text { no. }\end{array}$ & $\begin{array}{c}k \\
(\mathrm{~mL} / \mathrm{d})\end{array}$ & \multicolumn{1}{c}{$N$} & $\begin{array}{c}a \\
\left(\mathrm{~d}^{-1}\right)\end{array}$ & \multicolumn{1}{c}{$K$} & $\begin{array}{c}\gamma \\
(\mathrm{mL} / \mathrm{d})\end{array}$ \\
\hline 1 & $1.3452 \times 10^{-7}$ & 356 & 3.66 & 187.1 & $3.8199 \times 10^{-8}$ \\
2 & $8.1743 \times 10^{-6}$ & 7 & 1.8 & 41.1 & $4.2732 \times 10^{-7}$ \\
3 & $1.7437 \times 10^{-7}$ & 285 & 1.60 & 3.36 & $1.7475 \times 10^{-6}$ \\
4 & $1.7475 \times 10^{-7}$ & 253 & 1.02 & 31.3 & $1.0001 \times 10^{-8}$ \\
5 & $3.6064 \times 10^{-7}$ & 78 & 0.78 & 3.68 & $6.6248 \times 10^{-6}$ \\
6 & $1.0411 \times 10^{-7}$ & 501 & 2.49 & 2.20 & $1.0258 \times 10^{-5}$ \\
7 & $2.9845 \times 10^{-7}$ & 174 & 3.63 & 169.0 & $1.5195 \times 10^{-8}$ \\
8 & $2.2705 \times 10^{-7}$ & 211 & 2.00 & 95.2 & $6.4431 \times 10^{-8}$ \\
9 & $4.9959 \times 10^{-8}$ & 678 & 1.96 & 115.2 & $9.1986 \times 10^{-8}$ \\
10 & $6.3697 \times 10^{-7}$ & 41 & 1.75 & 0.39 & $3.08277 \times 10^{-6}$ \\
11 & $2.4603 \times 10^{-7}$ & 194 & 1.91 & 86.3 & $1.0660 \times 10^{-7}$ \\
12 & $2.3453 \times 10^{-6}$ & 17 & 4.18 & 92.3 & $1.2000 \times 10^{-8}$ \\
\hline
\end{tabular}

\section{Early Peaks in Postvaccination Plasma Viral Load}

Patients with relatively high baseline CD4 T-cell counts (patients 5, 9, 10,12), as well as those with high baseline viremia (patients 6,9) all had early peak viremia, at either 4 days (patients 5, 12) or 7 days (patients $6,9,10)$ postimmunization. This is reasonable because patients with a high baseline T-cell count or high viral load would be expected to have a larger population of target cells that could respond postimmunization. Thus, a vigorous early response by the immune system ensures an early peak in viremia.

The "viremia factor," or ratio of peak viremia to baseline viremia, given as $V_{\mathrm{p}} / V_{0}$ in Table 1 , was generally rather low for most patients, ranging between 2 and 4 (mean $=2.9)$ for 9 of 12 patients. Four remaining patients with unusually high "viremia factor" ratios, patients $3\left(V_{\mathrm{p}} / V_{\mathrm{o}}=7.5\right), 4\left(V_{\mathrm{p}} / V_{\mathrm{o}}=7.0\right), 10\left(V_{\mathrm{p}} / V_{\mathrm{o}}=\right.$ $35.7)$ and $12\left(V_{\mathrm{p}} / V_{\mathrm{o}}=9.3\right)$ also had early peaks, though in patients 4 and 12 this was a minor inflection or small peak followed by peak viremia at 21 and 28 days, respectively, whereas both patients 3 and 10 had single early peaks.

We were able to match well the amplitude and timing of peak viremia for patients 3, 6, and 10 (Fig. 2), all of whom had early peaks in viremia and were best-fit with 

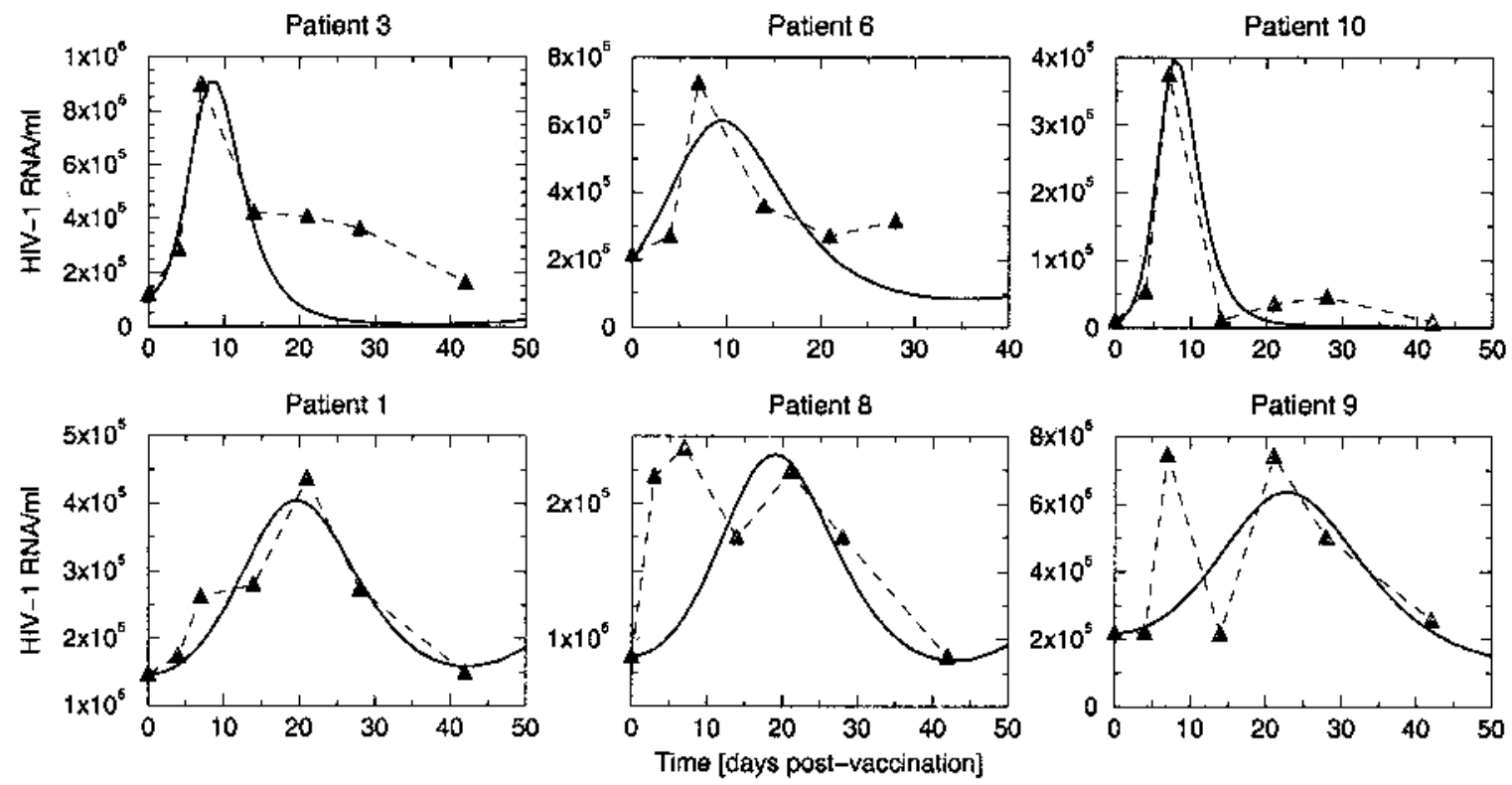

FIG. 2. Comparison of model (solid line) with data (triangles). Note that in some cases data sampling is such that the data curve is slightly asymmetric, and the true peak may not have been sampled. Patient 6 experienced a second very late peak (day 42) associated with known cavitary pneumonia, and the data was truncated at day 28.

relatively high antigen clearance rate constants, $\gamma$, in our simulations (Table 2).

\section{Double Peaks in Postvaccination Plasma Viral Load}

Over half of the patients studied had multiple peaks in viremia, though often the first peak might be classified as "minor." For patients with a minor early peak, usually at days 4 or 7, followed by a maximum in viral load at days 21 or 28 , we were able to fit the amplitude and timing of the maximum peak. Patients 1, 4, 7, 11, and 12 exemplify this behavior; results for patient 1 are shown in Figure 2. These patients were best-fit with $\gamma$ values that were low relative to those given for patients with true early peaks in viremia (Table 2). Patient 5 had very early viremia (day 4), followed by a second peak at day 21 . We were able to model the second peak, and from this obtained a relatively high antigen clearance constant.

Some patients experienced early and late peaks in viremia of equal or nearly equal amplitude. Of the patients with double peaks in viremia, our model generally fit the later peak at day 21 . These patients were assigned much lower clearance rates then were the patients who experienced single early peaks in viremia. However, in patients with double peaks (e.g., patients 8 and 9, Fig. 2) fits to the earlier peaks, unobtainable with this model, might require higher values of $\gamma$.

\section{T-cell-Antigen Interactions}

The rate of antigen induced T-cell activation/proliferation is given in the model by

$$
a\left(\frac{A}{A+K}\right)
$$

[equations 2 and 7]. Recall that the antigen concentration has been normalized, so that $A_{0}=1$ and $A \leq 1$. Thus, the initial rate of interaction, which is the highest rate since the antigen has not yet decayed, is given by

$$
a\left(\frac{1}{1+K}\right)
$$

For patients $1,2,4,7,8,9,11$, and $12, K \gg 1$ (Table 2). Thus, the denominator in the $\mathrm{T}$ cell activation term is approximately equal to $K$, and the initial activation/proliferation rate is

$$
\sim \frac{a}{K}
$$

Substituting in $K$ and $a$ values (Table 2) for these patients yields initial $a / K$ values ranging from $0.017 \mathrm{~d}^{-1}$ to $0.045 \mathrm{~d}^{-1}$ (mean value \pm sample standard deviation $\left.0.0275 \pm 0.011 \mathrm{~d}^{-1}\right)$ with most values clustered around $0.02 \mathrm{~d}^{-1}$. These patients all had low values for the T-cell activation/proliferation term, and late maxima (day 21: patients $1,4,7,8,9,11$; day 28: patients 2,12 ) in observed and modeled viremia. Note that for these patients, $a / K$ represents the average rate of T-cell proliferation in the presence of maximal antigen. Because not all $\mathrm{T}$ cells are expected to respond to tetanus toxoid, this $a / K$ value incorporates a factor proportional to the frac- 
tion of responding $\mathrm{T}$ cells. Thus, for example, if only $1 \%$ of $\mathrm{T}$ cells respond, then these cells on average divide with rate $2 \mathrm{~d}^{-1}$, i.e., on average every 12 hours, rather than the rate of $0.02 \mathrm{~d}^{-1}$ characteristic of all $\mathrm{T}$ cells.

Higher initial values of the activation term would increase the interaction between $\mathrm{T}$ cells and antigen $\mathrm{A}$, resulting in swifter activation of $\mathrm{T}$ cells and an earlier increase in virus. For patients $3,5,6$, and $10, A_{0}$ and $K$ were the same order of magnitude or within one order of magnitude in value, and the approximation for the interaction term shown above does not hold. In this case, the initial interaction term for these patients ranged from $0.21 \mathrm{~d}^{-1}$ to $4.5 \mathrm{~d}^{-1}$ (mean value $1.6 \pm 1.9 \mathrm{~d}^{-1}$ ), which is between one and two orders of magnitude greater than the interaction terms found for the prior group of "late peaking" patients. Patients 3, 6, and 10 all had early viremia, and relatively high values for the antigen clearance term $\gamma$. Note that patient 5 also had an early peak in observed viremia at day 4 that we could not fit with this model. Patient 5 had a second peak in viral load that we could fit, obtaining both a high initial interaction rate and a high antigen clearance rate. In patients 5 and 10, the large interaction term and antigen clearance rates may reflect relatively high baseline T-cell levels.

The parameter $a$ in our model represents the maximum rate of antigen-driven T-cell proliferation. From Table 2, the mean \pm sample standard deviation of $a=$ $2.2 \pm 1.1$. For a cell population dividing at rate $a, 1 / a$ is the average division time. Using the parameter estimates from Table 2, we find that the mean average division time is $0.56 \pm 0.30$ days or $13 \pm 7.2$ hours.

\section{Productively and Chronically Infected Cells}

Of concern for HIV-infected patients are any potentially long-lasting effects, aside from protective immunity to the vaccine antigen, associated with immunization. According to our model, the density of productively infected cells, $T^{*}$, tracks changes in viral load, peaking and falling at the same time. The effects of vaccination on longer-lived chronically infected cells, $C$, however, are more lingering. According to our model, the ratio of the maximum chronically infected cell level over baseline for all but one patient ranged from 1.6 to 6.1, with a mean of $2.7 \pm 1.3$, and the one outlier value of a 13 -fold increase in chronically infected cells (patient 10). At 28 days postimmunization, our simulations show these long-lived cells still elevated with respect to their initial (steady state) values, yielding increases of 1.4 to 3.0 over baseline, with a mean of $1.9 \pm 0.51$, disregarding the outlier value which is still high at 4.3 -fold above baseline and that of patient 12, who achieved a (modeled) post- immunization peak in chronically infected cells at day 32. At 42 days post immunization, chronically infected cells are still a factor of $1.3 \pm 0.46$ on average above baseline, again disregarding the outlier values of 1.9 (patient 10) and 6.1 (patient 12) above baseline. Indeed, focusing only on late-peaking patients, for whom infected and chronically infected cells are later to reach maximum, yields ratios of $2.3 \pm 0.65$ at maximum, $2.0 \pm$ 0.28 at 28 days, and $1.6 \pm 0.3$ at 42 days. By contrast, at 42 days postimmunization, productively infected cells for both patient populations have achieved or fallen below their baseline values. Modeled infected cell densities from patient 10 are shown plotted against plasma viral load (Fig. 3); note that chronically infected cells remain elevated long after infected cell populations have fallen to baseline values even in this early peaking patient. Thus while vaccination gives rise to short-term changes in viral load and productively infected cells, it may also produce longer lasting increases in the populations of chronically infected cells.

\section{DISCUSSION}

With a simple modification of a standard HIV infection model that takes into account antigen-driven proliferation of $\mathrm{T}$ cells, we are able to reproduce the general features of the postvaccination rise in viral load reported on by Stanley et al. (2). This implies that simply increasing the number of cells susceptible to infection by antigen activation, i.e., vaccination, can account for the modest rises in viral load observed by Stanley et al. (2) after tetanus booster vaccination. Interestingly, more than half of the patients in this study had double peaks in plasma HIV-1 RNA postvaccination. While assay variability might account for some of these peaks, in other cases the peaks were sufficiently large that this seems unlikely. For patients with double peaks we were only able to fit the later of the two peaks, which was often the larger peak. These patients were assigned lower antigen clearance rate constants, $\gamma$, than patients with early single peaks in viremia, which we also successfully modeled.

The fact that we could not approximate the earlier peaks in those patients with double peaks in viremia suggests that our model may lack some features present in the actual biology. For example, individuals vaccinated recently may have a larger and more robust memory cell response than individuals vaccinated a long time ago. The two peaks may represent an early memory response followed by a naive cell response. In individuals with little remaining memory, one may speculate that only the later naive response would be observed. However, because the two peaks frequently occur 14 days 


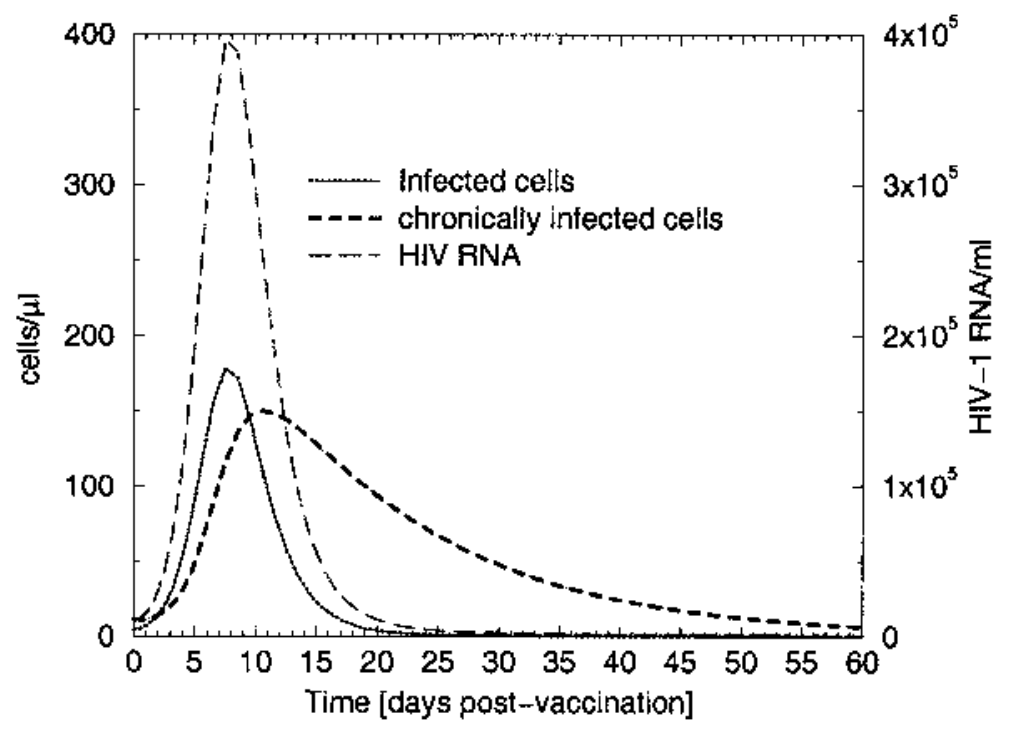

FIG. 3. Modeled postvaccination infected cell density and HIV-1 plasma viral load for patient 10 .

apart, this explanation would require a substantial difference in the time to activate naive and memory cell responses. Another possible explanation for double peaks is that the first peak is due to stimulation and infection of tetanus specific $\mathrm{T}$ cells, whereas the second peak is caused by the rise in viremia-stimulating HIV-specific $\mathrm{T}$ cells, or increasing the level of immune system activation, which in turn leads to an increase in target cell availability. We do not favor this explanation because our model, which follows the activation of $\mathrm{T}$ cells by antigen, had difficulty mimicking the fast rise in viremia needed to explain an early peak. One can speculate, then, that the early peak is not a result of the infection of vaccine-induced target cells, but rather a result of an effect such as the cytokine-induced enhancement of HIV transcription and release from infected cells, as has been seen in vitro with the use of tumor necrosis factor- $\alpha$ (4).

Despite the fact that the model failed to reproduce double peaks in viral load, the model could account for the timing and amplitude of the major peak in postvaccination plasma viremia. Because HIV-1, as observed in plasma, is produced by infected cells, the model suggests that levels of both productively infected and chronically infected cells also increase as a result of vaccination. Levels of latently infected cells presumably also increase, but in untreated individuals this population makes a minor contribution to plasma viral load. According to the model the increase in productively infected cells mirrored the observed changes in plasma viral load, and due to the short lifespan of these cells led to little long-term perturbation of this population. However, chronically infected cells have longer lifetimes and hence vaccination led to a larger period of increase in this population. Nevertheless, in all but two patients the increase was not large, with the maximum increase being 3.6-fold; in the most extreme case studied here, the increase was 13 -fold. However, by day 42 the chronically infected cell elevations were less than 2-fold in all but one patient. This, coupled with the fact that chronically infected cells generally produce only $1 \%$ to $7 \%$ of plasma virus (22), implies that the long-term impact of this change on disease progression should be small.

Acknowledgments: The authors thank Mario Ostrowski, Sharilyn Stanley, and Anthony Fauci for providing the primary data from the Stanley et al. (1995) study analyzed here. We also thank Rob J. De Boer for helpful discussions.

\section{REFERENCES}

1. Fauci AS. Multifactorial nature of human immunodeficiency virus disease: implications for therapy. Science 1993;262:1011-18.

2. Stanley SK, Ostrowski MA, Justement JS, et al. Effect of immunization with a common recall antigen on viral expression in patients infected with human immunodeficiency virus type $1 . N$ Engl J Med 1996;334:1222-30.

3. Wahl SM, Orenstein JM. Immune stimulation and HIV-1 replication. J Leukocyte Biol 1997;62:67-71.

4. Lawn SD, Butera ST, Folks TM. Contribution of immune activation to the pathogenesis and transmission of human immunodeficiency virus type 1 infection. Clin Microbiol Rev 2001;14:753-77.

5. Zack JA, Arrigo S, Weitsman SR, et al. HIV-1 entry into quiescent primary lymphocytes: molecular analysis reveals a labile, latent viral structure. Cell 1990:61:213-22.

6. Bukrinsky MI, Stanwick TL, Demspey MP, et al. Quiescent T lymphocytes as an inducible virus reservoir in HIV-1 infection. Science 1991;254:423-7.

7. Folks T, Kelly J, Benn S, et al. Susceptibility of normal human 
lymphocytes to infection with HTLV-III/LAV. J Immunol 1986; 136:4049-53.

8. Levacher M, Tallet $\mathrm{S}$, Dazza $\mathrm{M}$, et al. T cell activation marker evaluation in ARC patients treated with AZT: comparison with CD4+ lymphocyte count in non-progressors and progressors towards AIDS. Clin Exp Immunol 1990;81:177-82.

9. Giorgi JV, Detels R. T-cell subset alterations in HIV-infected homosexual men: NIAID multicenter AIDS cohort study. Clin Immunol Immunopathol 1989;52:10-18.

10. Medley GF, Anderson RM, Cox DR, et al. Incubation period of AIDS in patients infected via blood transfusion. Nature 1987;328: 719-21.

11. Bentwich Z, Kalkinovich A, Weisman, Z. Immune activation is a dominant factor in the pathogenesis of African AIDS. Immunol Today 1995;16:187-91.

12. Weissman D, Barker TD, Fauci AS. The efficiency of acute infection of CD4+ T cells is markedly enhanced in the setting of antigen-specific immune activation. J Exp Med 1996;183:687-92.

13. Staprans SI, Hamilton BL, Follansbee SE, et al. Activation of virus replication after vaccination of HIV-1-infected individuals. $J$ Exp Med 1995;182:1727-37.

14. O'Brien WA, Grovit-Ferbas K, Namazi A, et al. Human immunodeficiency virus type 1 replication can be increased in peripheral blood of seropositive patients after influenza vaccination. Blood 1995;86:1082-9.

15. Brichacek B, Swindells S, Janoff EN, et al. Increased plasma human immunodeficiency virus type 1 burden following antigenic challenge with pneumococcal vaccine. J Infect Dis 1996;174: $1191-9$.

16. Glesby MJ, Hoover DR, Farzadegan H, et al. The effects of influenza vaccination on human immunodeficiency virus type 1 load: a randomized, double-blind, placebo-controlled study. J Infect Dis 1996; 174:1332-6.

17. Rosok B, Voltersvik P, Bjerknes R, et al. Dynamics of HIV-1 replication following influenza vaccination of HIV+ individuals. Clin Exp Immunol 1996;104:203-7.

18. Tasker SA, O'Brien WA, Treanor JJ, et al. Effects of influenza vaccination in HIV-infected adults: a double-blind, placebocontrolled study. Vaccine 1998;16:1039-42.

19. Gunthard HE, Wong JK, Spina CA, et al. Effect of influenza vaccination on viral replication and immune response in persons infected with human immunodeficiency virus receiving potent antiretroviral therapy. J Infect Dis 2000;181:522-31.

20. Kolber MA, Gabr AH, De La Rosa A, et al. Genotypic analysis of plasma HIV-1 RNA after influenza vaccination of patients with previously undetectable viral loads. AIDS 2002;16:537-42.

21. Janoff EN, Tasker SA, Stevenson M, et al. Immune activation and virologic response to immunization in recent HIV type 1 seroconverters. AIDS Res Human Retroviruses 1999;15:837-45.

22. Perelson AS, Essunger P, Cao Y, et al. Decay characteristics of HIV-1 infected compartments during combination therapy. Nature, 1997;387:188-91.

23. Perelson AS, Neumann AU, Markowitz M, et al. HIV-1 Dynamics in vivo: virion clearance rate, infected cell life-span, and viral generation time. Science 1996;271:1582-6.

24. Nowak MA, May RM. The basic model of virus dynamics. In: Virus dynamics: mathematical principles of immunology and virology. New York: Oxford University Press, 2000:16-26.

25. McLean AR, Nowak MA. Models of interactions between HIV and other pathogens. J Theor Biol 1992;155:69-102.

26. Ferguson NM, deWolf F, Ghani AC, et al. Antigen-driven CD4+ T-cell and HIV-1 dynamics: residual viral replication under highly active antiretroviral therapy. Proc Natl Acad Sci USA 1999;96: 15167-72.

27. Chun T-W, Carruth L, Finzi D, et al. Quantification of latent tissue reservoirs and total body viral load in HIV-1 infection. Nature 1997;387:183-7.

28. Callaway D, Perelson AS. HIV-1 infection and low steady-state viral loads. Bull Math Biol 2002;64:29-64.

29. Mohri H, Bonhoeffer S, Monard S, et al. Rapid turnover of Tlymphocytes in SIV-infected rhesus macaques. Science 1988;279: $1223-7$

30. Mittler JE, Markowitz M, Ho DD, et al. Improved estimates for HIV-1 clearance rate and intracellular delay. AIDS 1999;13: $1415-17$.

\section{APPENDIX}

The steady state productively infected and chronically infected cell concentrations, $\bar{T}^{*}$, and $\bar{C}$, the infection rate constant, $k$, and burst size, $N$, may be calculated from the following steady state equations, derived by setting the left-hand sides of equations (2)-(5) to zero:

$$
\begin{aligned}
k & =\frac{\lambda-d \bar{T}}{\overline{V T}} \\
\bar{T}^{*} & =\frac{1}{\delta}(1-\alpha)(\lambda-d \bar{T}) \\
\bar{C} & =\frac{\alpha}{\mu}(\lambda-d \bar{T}) \\
N & =\frac{c \bar{V}-N_{c} \mu \bar{C}}{\delta \bar{T}^{*}}
\end{aligned}
$$

In addition, we impose the following conservation rule:

$$
\bar{T}_{T}=\bar{T}+\bar{T}^{*}+\bar{C},
$$

where $\bar{T}_{T}$ is the observed (total) T-cell count, comprising uninfected cells $\bar{T}$, productively infected cells $\bar{T}^{*}$, and chronically infected cells $\bar{C}$. From the above condition and equations (7) and (8), we derive the following relation for $\bar{T}$ :

$$
\bar{T}=\frac{\delta \mu \bar{T}_{T}-\lambda[(1-\alpha) \mu+\alpha \delta]}{\delta \mu-d[(1-\alpha) \mu+\alpha \delta]} .
$$

Parameters other than $k$ and $N$ were set as indicated in the text. Given these parameter choices, and the measured baseline $\mathrm{T}$ cell and viral load levels in the patients studied by Stanley et al. (2), this model generally yields 
larger steady state levels of chronically infected cells than of productively infected cells in these untreated patients (Table 2). In addition, for these parameter choices the denominator in equation 11 is positive, so that for the steady state level of target cells to be non-negative, i.e., for $\bar{T} \geq 0$, we require

$$
\bar{T}_{T} \geq \frac{\lambda[(1-\alpha) \mu+\alpha \delta]}{\delta \mu}=\lambda\left[\frac{(1-\alpha)}{\delta}+\frac{\alpha}{\mu}\right]
$$

or

\section{Rapid Communications}

Articles accepted as Rapid Communications will normally be published within 8 weeks of acceptance. When submitting a paper for consideration as a Rapid Communication, please adhere to the following guidelines:

- Submit your paper by fax or air express service to the appropriate editor with an accompanying letter stating why the paper merits special attention.

- All figures should be submitted by overnight express.

- The paper should not exceed 4 printed pages (approximately 12 double-spaced typewritten manuscript pages, including illustrations, tables, and references).

- The paper should include an abstract, key words, methods, results, discussion, and reference sections.

- The title page should include the corresponding author's telephone and fax numbers, and e-mail address.

- Authors will receive proofs of their article for review by fax and will be expected to return corrections by fax within 48 hours of receipt. Changes received after this deadline will not be accepted.

Papers that are not accepted as Rapid Communications may be resubmitted as full-length articles. 